\title{
A EXPERIÊNCIA DA VULNERABILIDADE DOS ENFERMEIROS NO CUIDADO EM TERAPIA INTENSIVA PEDIÁTRICA
}

Lidia Nunes Vieira ${ }^{1}$

Flávia Mendes de Sá ${ }^{1}$

Wilza Carla Spiri ${ }^{1}$

Maria Helena Borgato ${ }^{1}$

Cassiana Mendes Bertoncello Fontes ${ }^{1}$

Objetivo: Compreender as vulnerabilidades do processo de cuidar do enfermeiro em uma Unidade de Terapia Intensiva Pediátrica (UTIP). Metodologia: Estudo qualitativo, baseado no referencial teórico da ergologia e realizado em hospital de ensino público, no período de junho de 2016 a agosto de 2017. Foi utilizada a Análise de Conteúdo de Bardin para analisar as respostas dos enfermeiros assistenciais da UTIP em entrevistas individuais a partir de duas perguntas norteadoras: "Explique o que você compreende sobre vulnerabilidade" e "Como você experiência a vulnerabilidade no processo de cuidar do paciente internado na UTIP?" Resultados: Foram identificadas categorias e temas comuns e específicos referentes às perguntas norteadoras. Conclusões: Constatou-se a conceituação do termo vulnerabilidade e a análise das experiências vivenciadas pelos enfermeiros de diversas maneiras pelos indivíduos, o que possibilitou sua classificação em categorias de acordo com o tema pertencente. Descritores: Vulnerabilidade em saúde; Pesquisa qualitativa; Unidades de terapia intensiva pediátrica; Cuidados de enfermagem; Enfermagem.

\section{THE EXPERIENCE OF NURSES' VULNERABILITY IN PEDIATRIC INTENSIVE CARE}

Objective: To understand the vulnerabilities of the nurse care process in a Pediatric Intensive Care Unit (PICU). Methodology: Qualitative study based on the theoretical reference of ergology and carried out in a public teaching hospital from June 2016 to August 2017. The Content Analysis of Bardin was used to analyze the responses of nurses assisting the PICU in individual interviews from two guiding questions: "Explain what you understand about vulnerability" and "How do you experience vulnerability in the PICU process?" Results: Common and specific categories and themes have been identified for guiding questions. Conclusions: The conceptualization of the term vulnerability and the analysis of the experiences experienced by the nurses in different ways by the individuals was verified, which made possible their classification into categories according to the theme.

Descriptors : Health vulnerability; Qualitative research; Intensive care units pediatric; Nursing care; Nursing.

\section{LA EXPERIENCIA DE LA VULNERABILIDAD DE LAS ENFERMERAS EN CUIDADOS INTENSIVOS PEDIÁTRICOS}

Objetivo: comprender las vulnerabilidades del proceso de atención de enfermería en una unidad de cuidados intensivos pediátricos (UCIP). Metodología: estudio cualitativo basado en la referencia teórica de la ergología y realizado en un hospital público de enseñanza desde junio de 2016 hasta agosto de 2017. El análisis de contenido de Bardin se utilizó para analizar las respuestas de las enfermeras que asistieron a la UCIP en entrevistas individuales. a partir de dos preguntas guía: "Explique lo que entiende sobre la vulnerabilidad" y “¿Cómo experimenta la vulnerabilidad en el proceso de la UCIP?" Resultados: Se han identificado categorías y temas comunes y específicos para las preguntas guía. Conclusiones: se verificó la conceptualización del término vulnerabilidad y el análisis de las experiencias vividas por las enfermeras de diferentes maneras por los individuos, lo que hizo posible su clasificación en categorías según el tema.

Descriptores: Vulnerabilidad en salud; Investigación Cualitativa; Unidades de cuidado intensivo pediátrico; Atención de enfermeira; Enfermería. 


\section{INTRODUÇÃO}

O processo de tomada de decisões em ambiente crítico de Unidade de Terapia Intensiva Pediátrica (UTIP) envolve os princípios de autonomia e vulnerabilidade, pois permanece tenuamente contextualizado nas relações interpessoais, no desgaste gerado pelo estresse profissional e no risco inerente à assistência ao paciente $\mathrm{e}^{(1)}$.

O princípio da autonomia norteia a relação que existe entre os profissionais de saúde e os pacientes e contribui de maneira harmoniosa entre o sentir, o pensar e o agir.

O termo vulnerabilidade é polissêmico e implica sentido de desastre ou perigo, derivado do verbo no Latim vulnerare, significando "provocar uma injúria ou um dano". Nas pesquisas em saúde tal termo pode "designar suscetibilidade das pessoas e danos de saúde ${ }^{(2) "}$.

O conceito mais atual de vulnerabilidade consiste em demonstrar os potencais de adoecimento e não adoecimento do indivíduo sob determinadas condições ${ }^{(2)}$. Sendo assim, a vulnerabilidade pode ser definida como um estado sindrômico provocado por fatores ou condições diversas, e também de acordo com a percepção de cada indivíduo(3).

O referencial da Ergologia, desenvolvido pelo filósofo francês Yves Schwartz ${ }^{(2)}$, aborda conceitos fundamentais para a reflexão sobre os comportamentos humanos no ambiente de trabalho e suas relações. Na perspectiva ergológica de Schwartz é possivel perceber nas atividades de trabalho uma dimensão "micro" e outra "macro", o geral e o singular, onde a história está presente perpassando esses niveis da vida social(4).

A análise é realizada na medida de que é possivel levar em consideração o ponto de vista de quem trabalha e com a finalidade de investigar "o permanente debate de normas e de valores que renovam indefinidamente a atividade humana, concentrando-se sobre a relação que a pessoa estabelece com o meio no qual está engajada ${ }^{(5) "}$.

Em estudos realizados em UTIs pediátricas e neonatais constatou-se que a participação da família no cuidado fica limitada devido a separação da criança, o que gera conflitos, falta de diálogo e afastamento, evidenciando a vulnerabilidade do profissional ${ }^{(6-7)}$. Um desses trabalhos ${ }^{(7)}$ utilizou narrativas produzidas pelos enfermeiros para que eles pudessem refletir sobre sua prática e como cultivar o relacionamento com os pais.

Estudos apontam que as reinternações geram aumento da ansiedade da família, altos gastos de recursos materiais e tecnológicos, e promovem maior estresse profissional relacionado a demanda de leitos disponíveis ${ }^{(8-9)}$.

No que tange as questões éticas e legais, os estudos descreveram as aflições dos profissionais em relação a recomendação de não ressuscitação, tomada de decisão de suspensão de tratamento fútil, síndrome de abstinência neonatal e distanásia, bem como a falta de conhecimento, reflexão e evidencias científicas acerca desses temas ${ }^{(10-13)}$.

Os princípios éticos e morais que norteiam o cuidado de enfermagem intensivo são pilares que sustentam as decisões clínicas. Sendo assim, identificar como os enfermeiros descrevem suas experiências e as vulnerabilidades a que se submetem no processo de cuidar, podem trazer contribuições para a prática baseada em evidências éticas e nos princípios da beneficência e não maleficência.

Dessa maneira esse estudo tem por finalidade descrever a experiência sobre a vulnerbilidade dos enfermeiros de terapia intensiva pediátrica de um hospital público de ensino.

O objetivo do estudo foi compreender as vulnerabilidades do processo de cuidar do enfermeiro na UTIP.

\section{METODOLOGIA}

\section{Referencial teórico-metodológico}

Estudo de abordagem qualitativa por meio da Análise de Conteúdo de Bardin ${ }^{(14)}$, na vertente temática, como técnica para identificar os significados, e a análise lexical para os significantes da realidade além da mensagem.

\section{Participantes da pesquisa}

A amostra foi constituída por nove enfermeiros assistenciais escalados na UTIP, independentemente, do turno, da carga horária semanal, do tipo de vínculo empregatício, do cargo e função e cobertura de absenteísmo. Os participantes foram incluídos após aceitarem e assinarem o Termo de Consentimento Livre e Esclarecido.

\section{Local de estudo}

A unidade pertence a um hospital público de ensino, possui sete leitos para internação da faixa etária de 30 dias a 15 anos de idade. As pesquisadoras possuem vínculo de ensino e pesquisa na UTIP devido ao convênio de atividades curriculares do Curso de Graduação de Enfermagem.

\section{Coleta dos dados}

Realizou-se uma entrevista individual com os participantes em sala privativa da UTIP, agendadas previamente, com duração média de 20 minutos, fora do horário de trabalho, pelas pesquisadoras que registraram as respostas referentes às duas perguntas norteadoras: "Explique o que você compreende sobre vulnerabilidade" e "Como você experencia a vulnerabilidade no processo de cuidar do paciente internado na UTIP?" 
Análise dos dados

As unidades de falas foram analisadas e descritas por classes e categorias permitindo evidenciar os temas que inferem sobre a realidade. A discussão dos dados foi realizada à luz do referencial da ergologia.

Procedimentos éticos

O estudo obteve aprovação do Comitê de Ética em Pesquisa (CEP) da instituição. Após esclarecer os objetivos, garantia de anonimato e os benefícios do estudo, em observância à legislação sobre pesquisa envolvendo seres humanos, o Termo de Consentimento Livre e Esclarecido (TCLE) foi aplicado em duas vias, uma delas entregue a pesquisadora.

\section{RESULTADOS}

Os resultados foram descritos e analisados a partir das respostas às duas perguntas norteadoras e identificadas as categorias representativas dos conceitos de vulnerabilidades, porém alguns desses conceitos foram comuns.

As categorias advindas da primeira pergunta foram: exposição emocional, física e social do enfermeiro; fragilidade da criança; risco de acontecer evento e estar exposta a qualquer coisa e ao ambiente; infecção; acidentes biológicos; eventos adversos; riscos físico, biológico e emocional.

As categorias relacionadas à segunda pergunta foram as vulnerabilidades: de mão dupla; aos sentimentos e emoções; cuidado paliativo; família; impotência; exposição ao ambiente; falta de material; acidentes pérfuro cortantes; falta autonomia e cuidado centrado no tratamento médico.

A seguir, os dois temas identificados e que são comuns às duas perguntas norteadoras foram: "Conceito de vulnerabilidade relacionado aos riscos e acidentes de trabalho" e o "Conceito de vulnerabilidade relacionado aos problemas organizacionais/ administrativos".

Conceito de vulnerabilidade relacionado aos riscos e acidentes de trabalho

Neste tema os participantes destacam sua perspectiva de vulnerabilidade relacionando-a ao ambiente laboral e seus riscos:

Os riscos biológicos: contato secreções, pacientes com diversas patologias, leque grande. Tem crianças maiores.

(El)

Vulnerável a acidentes biológicos; eventos adversos. (E5)

Existe uma vulnerabilidade dupla, por exemplo a perfuração com a agulha. (E7)

Estar vulneráuel as doenças dos pacientes, a se contaminar com secreções. (E9)
Conceito de vulnerabilidade relacionado aos problemas organizacionais/administrativos

A percepção do enfermeiro que relaciona sua vulnerabilidade ao contexto no qual está inserido:

Principalmente com relação à prescrição medica, devido ter muito residente, por ser hospital universitário, no sentido de induzir ao erro de medicação; mesmo questionando no sentido de dosagens. (E5)

Otrabalho multiprofissional percebido como determinante de vulnerabilidades:

Tomada de decisão em UTI que depende de outros profissionais e tem que analisar. (E2)

Erros médicos, prescrição medica, erros da enfermagem. (E5)

A falta de recursos humanos e materiais é um tema recorrente nas falas:

Os profissionais trabalham muito, difícil $R H$, sobrecarga de trabalho. (El)

Dimensionamento inadequado, falta pessoal, falta material, mascara em precauções por gotículas. Exposição à radiação. (E7)

Dos temas que emergiram da análise da primeira pergunta temos:

O conceito de vulnerabilidade sob o aspecto da polissemia indicando susceptibilidade a algo.

Você está suscetivel a alguma coisa. (E1)

Quando está em risco, a frequência com que pode acontecer um evento. (E6)

Exposição, o quanto exposto a gente está, várias coisas, parte emocional, espiritual, física. (E7)

Estar exposto a algo, de uma maneira indireta. (E8)

Algo que seja fácil e tenha probabilidade de acontecer.

Nota-se que as entrevistadas definiram sua compreensão de vulnerabilidade relacionando-a à suscetibilidade e exposição física e emocional.

Conceito de vulnerabilidade relacionado à chance de ocorrência de um evento ou doença.

No que tange a vulnerabilidade e ocorrência de doenças, 
as falas dos participantes descrevem:

Está sujeito ao incerto, algo incerto, doença. (E2)

O que as crianças estão vulneráveis a ter: infecção no sangue, urinária, pneumonia devido infecção pulmonar.

Conceito de vulnerabilidade relacionado ao envolvimento emocional com paciente e família.

A fala expressa a intensificação da carga emocional na UTIP

Suscetivel às emoções, aos sentimentos, envolvimento pessoal com a familia e o paciente. Isso afeta a minha pessoa. (E4)

A partir da análise dos conteúdos da segunda pergunta surgiram os seguintes temas:

Vulnerabilidade experienciada através dos processos comunicativos dentro da equipe de saúde

Os participantes desvelam esse tema nas seguintes falas:

Equipe médica não escuta, não valoriza a equipe de enfermagem, muitas vezes a gente avisa, comunica e eles não ouvem, independente de que membro da equipe. Às vezes dá um pouquinho mais de ouvido para o técnico de enfermagem. (E5)

Parte do estresse, independente da sobrecarga, ter dó, discute com o médico se precisa fazer o procedimento novamente. (E7)

Vulnerabilidade experienciada pelos processos dolorosos dos pacientes

Nesse tema revela-se que os processos dolorosos vivenciados pelos profissionais são vulnerabilidades as quais os profissionais estão expostos. As falas expressam: Estória do paciente: dar mais atenção, ficar abalada com a criança, é mais emotivo, se apega. (E3)

Não é fácil ver uma criança morrer, ou sair daqui neuropata, ainda mais que tenho filho, tem que ter muito amor. (E9)

Vulnerabilidade experienciada no processo de cuidar da enfermagem

Os profissionais necessitam desenvolver competências clínicas, pois essas interferem na situação de saúde do paciente, como se observa na unidade de fala a seguir:

A destreza, sensibilidade motivacional tem que estar alinhada para conseguir credibilidade e atuar com ação. Procurar um suporte para questão de atuar. Na dúvida eu não faço nada, tem que ter confiança no que estiver fazendo. No tratamento com as crianças entubadas, sedadas, será que a conduta é adequada, é certa? (E2)

Em outra fala, uma enfermeira relata como o ambiente da UTI pediátrica exige que suas competências clínicas sejam individualizadas:

É um lugar crítico, as crianças vêm debilitadas, diferente de outros setores, são mais frágeis. Isso traz mais cuidado para evitar de ter outros problemas, específico para cada um, mais atenção, mais higiene. Para não evoluir para outras patologias, doença secundária devido a fragilidade. (E3)

Vulnerabilidade experienciada através da fragilidade do paciente.

O sentimento de impotência foi citado nos discursos, possibilitando notar a presença de fatores emocionais em suas experiências:

Sensação de que poderia ter feito mais, arrependimento sobre ações inevitáveis, sensação de impotência, autocobrança. (E4)

Emoções do cuidado paliativo, vê a criança definhando diariamente, não tem o que fazer, a criança pede ajuda e o enfermeiro não pode fazer nada, o olhar de desespero da criança. (E5)

Na UTI pediátrica o paciente é submisso aos cuidados, ele não pode escolher o que, o horário. (E6)

Vulnerabilidade experienciada através do vínculo entre a familia/paciente e equipe de saúde.

A vulnerabilidade foi relatada através da empatia pelo sofrimento da família e paciente:

Vivenciar perdas de familiares das crianças, óbitos das próprias crianças, crianças com câncer relatando dor, crianças em situações de ausência familiar e negligência. (E4)

As enfermeiras também relatam como experienciam sua vulnerabilidade através das reações dos pais diante de más notícias:

Enfermeira fica vulnerável as reações emocionais da família diante da doença da criança e pela fase de negação. (E5)

Tem os pais, você não sabe como eles vão reagir e às vezes não é muito agradável essa reação, então você está vulnerável. (E7) 


\section{DISCUSSÃO}

As vivências dos participantes na UTIP revelaram as vulnerabilidades que esses profissionais vivenciam no cotidiano de seu trabalho, principalmente no que se refere ao cuidar, essência do trabalho da enfermagem.

Assim, corrobora-se com a literatura que porconsequência da necessidade de realização de procedimentos, os profissionais da enfermagem são os que possuem maior contato com os pacientes, expondo-os diariamente a fatores que comprometem sua segurança, como riscos biológicos, físicos, químicos, ergonômicos e ocupacionais ${ }^{(15)}$.

Os cuidados intensivos propiciam maior exposição aos microrganismos presentes nas secreções e no ambiente, ocasionando contaminações decorrentes da manipulação de instrumentos perfurocortantes, contato com agentes lesivos, sangue e fluidos corpóreos, além da exposição à radiação, por imprudência ou falta de recursos, substâncias químicas como medicamentos ou produtos para higienização de materiais e cuidados que requerem esforços físicos, como o posicionamento e locomoção de pacientes, prejudiciais à ergonomia do profissional(15). Além da exposição implícita do ambiente, a complexidade dos pacientes implica em uma maior suscetibilidade à disseminação e contaminação de patógenos, acarretando nas infecções relacionadas à assistência à saúde. Também considera-se a carga de trabalho e a proporção de profissionais para pacientes como fatores de disseminação(16).

Um entrevistado cita o erro de medicação e relaciona-o ao ambiente de hospital-escola, onde é comum a atuação de profissionais em formação e erros podem ocorrer com maior frequência, por isso é importante que a cultura organizacional desse tipo de instituição promova a segurança do trabalhador e monitoramento de suas ações de maneira educativa e não punitiva, tendo em vista a prevenção de erros-cascata, no qual o profissional da enfermagem atribui vulnerabilidade. Um estudo realizado com enfermeiros de uma UTI admite a influência do ambiente na hipótese de que erros não advêm somente dos profissionais, mas possuem origens sociais e institucionais. A omissão ocorre principalmente, quando envolve mais pessoas ou equipes, sendo relacionada à falta de discernimento do profissional de que a responsabilidade diante dos seus erros supõe o reconhecimento das próprias vulnerabilidades ${ }^{(17)}$.

A falta de recursos humanos e materiais é reconhecida pelos participantes como vulnerabilidade, estudo indica que quando o profissional não dispõe de todas as condições necessárias para prestar uma assistência segura pode ocasionar a exaustão e estresse, considerando-se também que a falta de materiais proporciona risco a sua segurança e a do paciente. Um ambiente de trabalho deve dispor de capacidade de organização, planejamento de recursos materiais e tecnológicos de qualidade, competência clínica demonstrando necessidade de desenvolvimento de suporte organizacional e controle que favoreça a satisfação profissional e o desempenho(18,19).

Os relatos exemplificam como os participantes vivenciam a comunicação inadequada e o relacionamento entre equipes. $O$ distanciamento entre os profissionais da saúde tem se tornado comum e a falta de diálogo resulta em compartimentalização do cuidado, gerando interferências negativas ao paciente e insatisfação profissional(18). Embora haja estratégias de comunicação em uma unidade, deve-se garantir que estas sejam eficazes para todos os profissionais, o que pode auxiliar, inclusive, em situações de mudanças bruscas de rotina ou ambiente, corroborando com transições menos conflituosas ${ }^{(20)}$.

O reconhecimento da competência clínica é citado pelos entrevistados, é importante em UTI e pode ser compreendida como a aplicação das habilidades técnicas e comunicativas, conhecimento e raciocínio científicos, emoções e valores no ambiente clínico, que influenciam na qualidade do cuidado. A capacidade de julgamento, administração e avaliação adequadas são habilidades que o enfermeiro desenvolve em sua formação associada ao trabalho no ambiente clínico(21).

O envolvimento emocional é um componente da exposição referida, comum entre os profissionais que possuem uma "personalidade do trabalho"(22) para suprimir seus sentimentos com intuito de passar segurança e qualidade.

As falas refletem o empenho que os enfermeiros colocam em suas ações para mostrar compromisso e responsabilidade perante à criança e seus pais, e a frustração em casos de piora ou óbito. Na UTIP os enfermeiros são expectadores dos processos dolorosos, da separação entre a criança e seus familiares, dos medos, angústias, ansiedade, da dor da perda, além de presenciar o abandono familiar e negligência.

Um estudo constatou que os enfermeiros de uma UTI neonatal dispendem tempo e energia para manter uma postura profissional de competência, sem demonstrar o desgaste emocional ocasionado por fatores que envolvem a vida particular do enfermeiro, como em profissionais em idade fértil e/ou mães e pais que associam a criança doente ao próprio filho, resultando em sentimentos de empatia pela criança e familiares. Também às alterações emocionais desencadeadas pelas demandas dos pais, que sentem-se ansiosos em relação ao prognostico da criança e interação com a equipe e ambiente. Por isso, os pais necessitam de uma atenção com direito a informações que sejam facilmente compreendidas, por isso dispende-se de um preparo emocional o enfermeiro ter que informar más notícias que vão de encontro a expectativa dos pais, e ainda colocar-se 
disponivel para ajuda-los no enfrentamento(22, 23). Além dos demais estressores que advém da organização da unidade e instituição.

Neste cenário, os enfermeiros ficam mais suscetíveis aos sentimentos de corresponsabilidade e impotência, mesmo ciente de que o processo de cura ou melhora não depende unicamente de suas ações, consequentemente, optam frequentemente por camuflar essas emoções ${ }^{(22)}$.

\section{Limitações do estudo}

A abordagem da vulnerabilidade sobre a ótica da ergologia tem pouca utilização no contexto da saúde o que torna o referencial teórico escasso. A utilização da pesquisa qualitativa não permite a generalização dos dados, no entanto, o aprofundamento da temática é essencial para ampliar o conhecimento sobre este.

\section{Contribuições do estudo para a prática}

Este estudo proporcionou aos pesquisadores conhecer os fenômenos das vulnerabilidades sobre o processo de cuidar em uma UTIP e possibilitou identificar fortalezas, fragilidades e desafios a partir da compreensão das experiências dos enfermeiros. A atribuição dos significados vislumbrou vulnerabilidades advindas de todos os aspectos, além do físico e do biológico; e o referencial teórico permitiu um olhar relacional para além do cenário, do binômio paciente-família e do cuidar.

\section{CONCLUSÃO}

Ao buscar compreender as vulnerabilidades dos enfermeiros da UTIP constatou-sea conceituação polissêmica do termo o que retrata a singularidade com que cada profissional percebe suas vivências. $O$ estudo proporcionou identificar dois temas em comum no processo de cuidar do enfermeiro assistencial em terapia intensiva pediátrica, que emergiram da análise das perguntas norteadoras, demonstrando a forte relação entre vulnerabilidade e riscos biológicos e as questões do conceito de vulnerabilidade relacionados aos problemas organizacionais e administrativos institucionais. Observa-se, portanto, como essas questões conceituais podem afetar a performance do enfermeiro.

\section{Contribuição dos autores}

Lídia Nunes Vieira; Flávia Mendes de Sá e Cassiana Mendes Bertoncello Fontes: concepção;análise e interpretação dos dados; redação do artigo; revisão crítica e revisão final.

Wilza Carla Spiri e Maria Helena Borgato: revisão crítica e revisão final. 


\section{REFERÊNCIAS}

1. Béranger A, Boize P, Viallard ML. The practices of withdrawing artificial nutrition and hydration in the neonatal intensive care unit: a preliminary study. Arch Pediatr [Internet]. 2014 [cited 2016 Jun 05]:21(2):170-6. Available from: https://ac.els-cdn.com/ S0929693X13005885/1-s2.0-S0929693X13005885-main.pdf? tid=9b986c76-2e7f-4ale-bf5a-67b78e87f156\&acdnat $=1527516486$ ebcffb90765ecd0549d6efaeb6ea7ec6.doi: 10.1016/j.arcped.2013.11.006.

2.Nichiata LYI, Bertolozzi MR, Takahashi RT, Fracolli LA. A utilização do conceito "vulnerabilidade" pela enfermagem. Rev Lat Am Enfermagem [Internet]. 2008 [cited 2016 Jun 05];16(5):1-7. Available from: http://www. scielo.br/pdf/rlae/vl6n5/pt_20.

3. Hardin SR. Vulnerability of older patients in critical care Crit Care Nurse [Internet]. 2015 [cited 2018 Mai 10];35(3):5560. Available from: http://citeseerx.ist.psu.edu/viewdoc/ download?doi=10.1.1.1018.7344\&rep=replEtype=pdf. doi: http://dx.doi. org/10.4037/ccn2015995.

4. Oliveira S, Alvarez D, Brito J. A dimensão gestionária do trabalho: aspectos da atividade de cuidado. Cienc Saude Colet [Internet]. 2013 [cited 2018 Mai 10]:18(6):1581-9. Availalbe from: http://www.scielo.br/pdf/csc/ v18n6/10.pdf. doi: http://dx.doi.org/10.1590/S1413-81232013000600010.

5.Holz EB, Bianco MF. Ergologia: uma abordagem possivel para os estudos organizacionais sobre trabalho. Cad EBAPE [Internet]. 2014 [cited 2016 Jun 09]:12(Esp):495-512. Available from: http://www.scielo.br/pdf/ cebape/v12nspe/07.pdf. doi: http://dx.doi.org/10.1590/1679-39519106.

6.Côa TF, Pettengill MAM. A experiência de vulnerabilidade da familia da criança hospitalizada em Unidade de Cuidados Intensivos Pediátricos. Rev Esc Enferm USP [Internet]. 2011 [cited 2016 Jun 09]:45(4):825-32. Available from: http://www.revistas.usp.br/reeusp/article/view/40771/44107.

7.Ringham C. Narratives and embodied knowing in the NICU. Neonatal Netw [Internet]. 2012 [cited 2016 Jun 10]:31(1):16-9. doi: 10.1891/07300832.31.1.16

8.Catlin AJ. Extremely long hospitalizations of newborns in the United States: data, descriptions, dilemmas. J Perinatol [Internet]. 2006 [cited 2016 Jun 11];26(12):742-8. Available from: https://www.nature.com/ articles/7211617.pdf. doi: 10.1038/sj.pj.7211617.

9. Wightman A, Largent E, Del Beccaro M, Lantos JD. Who should get the last PICU bed? Pediatrics [Internet]. 2014 [cited 2016 Jun 10]:133(5):90712. Available from: http://pediatrics.aappublications.org/content/ pediatrics/133/5/907.full.pdf. doi: 10.1542/peds.2013-3369.

10.Huang LC, Chen CH, Liu HL, Lee HY, Peng NH, Wang TM, et al. The attitudes of neonatal professionals towards end-of-life decisionmaking for dying infants in Taiwan. J Med Ethics [Internet]. 2013 [cited 2016 Jun 10];39(6):382-6. Available from: http://jme.bmj.com/content/ medethics/39/6/382.full.pdf. doi: 10.1136/medethics-2011-100428.

11.Maguire D, Webb M, Passmore D, Cline G. NICU nurses' lived experience: caring for infants with neonatal abstinence syndrome. Adv Neonatal Care [Internet]. 2012 [cited 2016 Jun 10];12(5):281-5. Available from: https://insights.ovid.com/pubmed?pmid=22964603. doi: 10.1097/ ANC.ObO13e3182677bcl.

12.Akpinar A, Senses MO, Er RA. Attitudes to end-of-life decisions in paediatric intensive care. Nurs Ethics [Internet]. 2009 [cited 2016 Jun 17]:16(1):83-92. Available from: http://journals.sagepub.com/doi/ pdf/10.1177/0969733008097994. doi: 10.1177/0969733008097994.
13.Santana JCB, Rigueira ACM, Dutra BD. Distanásia: reflexões sobre até quando prolongar a vida em uma unidade de terapia intensiva na percepção dos enfermeiros. Bioethikos [Internet]. 2010 [cited 2016 Jul 03]:4(4):402-11.

14.Bardin L. Análise de conteúdo. Lisboa: Edições 70; 2009.

15.Albuquerque SGE, Castro RD, Ferreira GLS, Oliveira KL. Fatores de risco à segurança do enfermeiro na unidade de terapia intensiva de um Hospital Geral. Rev Bras Cienc Saude. 2015;19(2):135-42. doi: 10.4034/ RBCS.2015.19.02.08.

16.Nogueira LS, Rebustini RELF, Poveda VB, Silva RCG, Barbosa RL, Oliveira EM, et al. Carga de trabalho de enfermagem: preditor de infecção relacionada à assistência à saúde na terapia intensiva? Rev Esc Enferm USP [Internet]. 2015 [cited 2017 Feb 10]:49(Esp):36-42. Available from: http://www.scielo.br/pdf/reeusp/v49nspe/1980-220X-reeusp-49spe-0036.pdf. doi: 10.1590/S0080-623420150000700006.

17.Coli RCP. Anjos MF, Pereira LL. Postura dos enfermeiros de uma unidade de terapia intensiva frente ao erro: uma abordagem à luz dos referenciais bioéticos. Rev Lat Am Enfermagem [Internet]. 2010 [cited 2017 Jan 22];18(3):27-33. Available from: http://www.scielo.br/pdf/rlae/ v18n3/pt_05.pdf

18.Coronetti A, Nascimento ERP, Barra DCC, Martins JJ. O estresse da equipe de enfermagem na unidade de terapia intensiva: o enfermeiro como mediador. ACM Arq Catarin Med [Internet]. 2006 [cited 2017 Mai 23];35(4):36-43. Available from: http://www.acm.org.br/revista/pdf/ artigos/394.pdf.

19. Oliveira EM, Barbosa RL, Andolhe R, Eiras FRC. Padilha KG. Nursing practice environment and work satisfaction in critical units. Rev Bras Enferm [Internet]. 2017 [cited 2018 Mai 23];70(1):73-80. Available from: http://www.scielo.br/pdf/reben/v70nl/en_0034-7167reben-70-01-0079.pdf. doi: http://dx.doi.org/10.1590/0034-7167-20160211 .

20.Lin FF, Foster M, Chaboyer W, Marshall A. Relocating an intensive care unit: an exploratory qualitative study. Aust Crit Care [Internet] 2016 [cited 2018 Mai 16];29(2):55-60. Available from: https://ac.els-cdn $\mathrm{com} / \mathrm{S} 1036731415001046 / 1-\mathrm{s} 2.0-$ S1036731415001046-main.pdf? tid=6b487f62-1452-40a7-947b-b9913ed408ab\&acdnat=1527511494_ 26efba445889713d5e37dde451f9313b. doi: http://dx.doi.org/10.1016/j. aucc.2015.09.001.

21.Soroush F, Boroujeni AZ, Namnabati M. The relationship between nurses clinical competence and burnout in neonatal intensive care units. Iran J Nurs Midwifery Res. 2016:21(4):424-9. doi: 10.4103/1735-9066.185596.

22.Lizza RC. The need to nurse the nurse: emotional labor in neonatal intensive care. Qual Health Res [Internet]. 2014 [cited 2017 Out 14]:24(5):615-28. Available from: http://journals.sagepub.com/doi/ pdf/10.1177/1049732314528810. doi: 10.1177/1049732314528810.

23. Majdalani MN, Doumit MAA, Rahi AC. The lived experience of parents of children admitted to the pediatric intensive care unit in Lebanon. Int J Nurs Stud [Internet]. 2014 [cited 2018 Mai 23];51(2):217-25. Available from: https://www.journalofnursingstudies.com/article/SOO2O7489(13)00182-X/pdf. doi: http://dx.doi.org/10.1016/j.jnurstu.2013.06.001. 